\title{
Kinetic Roughening and Material Optical Properties Influence on Van der Waals/Casimir Forces
}

\author{
P.J. VAN ZwOl AND G. PAlasantzas* \\ Materials innovation institute M2i and Zernike Institute for Advanced Materials \\ University of Groningen, 9747 AG Groningen, Netherlands
}

\begin{abstract}
Atomic force microscopy measurements and force theory calculations using the Lifshitz theory show that van der Waals/Casimir dispersive forces have a strong dependence on surface roughness and material optical properties. It is found that at separations below $100 \mathrm{~nm}$ the roughness effect is manifested through a strong deviation from the normal scaling of the force with separation distance. Moreover, knowledge of precise optical properties of metals is shown to be very important for accurate force predictions rather than referring to idealized defect free material models. Finally, we compare the van der Waals/Casimir forces to capillary adhesive forces in order to illustrate their significance in stiction problems.
\end{abstract}

PACS numbers: 68.55.Jk, 68.37.Ps, 85.85. $+\mathrm{j}, 78.68 .+\mathrm{m}, 85.85 .+\mathrm{j}, 68.55 .-\mathrm{a}, 47.55 . \mathrm{nb}$

\section{Introduction}

When the proximity between material objects becomes of the order of nanometers up to a few microns, a regime is entered where forces quantum mechanical in nature, namely, van der Waals (vdW) and Casimir (retarded $\mathrm{vdW}$ ) forces, become operative [1]. Historically, the Casimir force has been considered to result from the perturbation of vacuum zero point fluctuations (ZPF) by conducting plates. Indeed, quantum theory predicts that the vacuum of space in the universe is filled with low-energy electromagnetic waves, random in phase and amplitude and propagating in all possible directions [1]. Because of its relatively short range, the Casimir force is now starting to take on technological importance in the operation of micro/nano-electromechanical systems (MEMS/NEMS) at separations less than $200 \mathrm{~nm}$, e.g., micro oscillator devices, micro/nano switches or actuators [2]. Moreover, from a fundamental point of view the Casimir force plays important role in the search of hypothetical forces beyond the standard model $[2,3]$.

Although the first high accuracy measurements by Lamoreaux [4] initiated further detailed investigations at close separations including atomic force microscopy (AFM; see Fig. 1) [5, 6], it remains still a challenge of how well we understand the concept of zero point vacuum fluctuations. Indeed, their energy density is given by $[1,4]$

\footnotetext{
* corresponding author; e-mail: G.Palasantzas@rug.nl
}

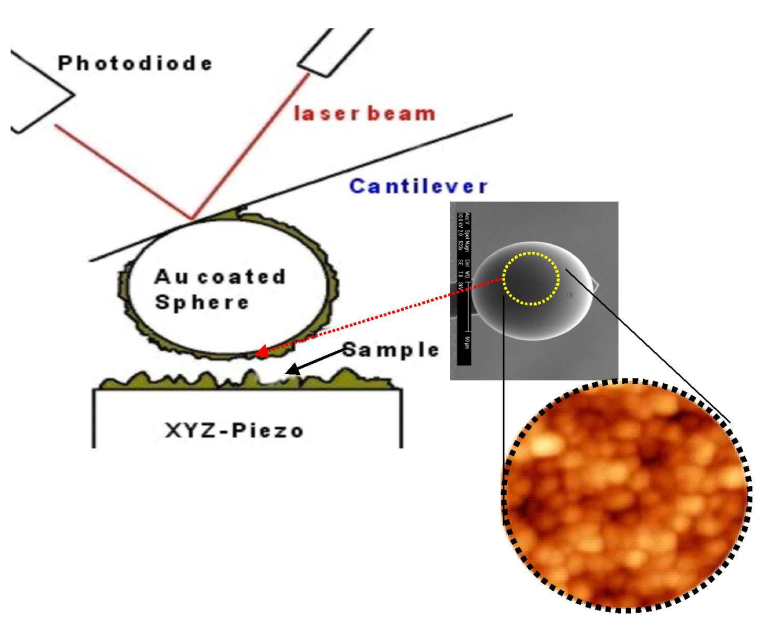

Fig. 1. AFM set-up for force measurements. The sphere is usually coated by $\mathrm{Au}$ (typically $\sim 100 \mathrm{~nm}$ thick).

$$
\rho_{\mathrm{ZPF}}=\frac{\hbar c}{2 \pi} \int_{0}^{\mathrm{K}_{\max }} q^{3} \mathrm{~d} q
$$

with $K_{\max }=2 \pi / L_{\mathrm{m}}$ and $L_{\mathrm{m}}$ a minimum length scale of vacuum fluctuations. If we consider as a minimum length scale $L_{\mathrm{m}}$ the Planck length $L_{\mathrm{Pl}}=\sqrt{\hbar G / c^{3}}=$ $1.6 \times 10^{-33} \mathrm{~cm}$ (the space itself is thought to break up into a kind of quantum foam beyond this length) with $G$ the Gravitational constant, $\hbar$ the Planck's constant, and $c$ the velocity of light), then we obtain $\rho_{\mathrm{ZPF}}=$ $10^{95} \mathrm{~g} / \mathrm{cm}^{3}$ [4]. If we consider as a more reasonable value for $L_{\mathrm{m}}$ the electron radius or $r_{\mathrm{e}}=2.82 \times 10^{-13} \mathrm{~cm}$ then we obtain $\rho_{\mathrm{ZPF}}=10^{14} \mathrm{~g} / \mathrm{cm}^{3}$ [4]. Nevertheless, both values of the energy density $\rho_{\mathrm{ZPF}}$ are still very large. Indeed, 
dark energy density estimations yield an experimental density value $\rho_{\text {Dark }} \approx 10^{-29} \mathrm{~g} / \mathrm{cm}$ [4]. If we compare it to zero point fluctuations of the vacuum we have an extremely large discrepancy $\rho_{\mathrm{ZPF}} / \rho_{\text {Dark }} \approx 10^{43} \rightarrow 10^{124}$ representing the biggest discrepancy in physics [4]. If on the other hand we fix $L_{\mathrm{m}}$ so that $\rho_{\mathrm{ZPF}} \approx \rho_{\text {Dark }}$ then the measured Casimir force would be much smaller than actual values obtained experimentally [4]. This issue remains still under investigation and it will lead research deep into the future in combination with challenges to Newtonian gravitation [3].

In any case, van der Waals/Casimir forces between real materials can be strongly influenced by surface morphology and material optical properties as it will be shown in the following. These are important issues if the influence of these dispersive forces in MEMS/NEMS is under consideration. Moreover, comparison of the dispersive forces in real materials to other forces, as for example capillary forces, that influence device motion, is also necessary.

\section{Lifshitz theory and real materials}

The van der Waals/Casimir force between real materials is calculated using the Lifshitz theory [6]. For real parallel flat mirrors of area A, separation distance $L$, and reflection coefficient $r(\Phi)$ (with $\Phi$ the imaginary frequency of the electromagnetic wave), the van der Waals/ Casimir energy $E_{\mathrm{pp}}$ is given by (assuming absolute temperature $T=0 \mathrm{~K}$ and short separations, e.g., $L<1 \mu \mathrm{m}$, where thermal corrections are negligible) [4]

$$
\begin{aligned}
& E_{\mathrm{pp}}= \\
& \quad-\hbar A \sum_{v} \int \frac{\mathrm{d}^{2} k}{4 \pi^{2}} \int_{0}^{\infty} \frac{\mathrm{d} \Phi}{2 \pi} \ln \left[1-r^{v}(k, \Phi)^{2} \mathrm{e}^{-2 \kappa L}\right] .
\end{aligned}
$$

The index $v$ denotes the sum over the $s$ and $p$ polarization. Since for experimental force measurements by AFM a sphere-plate geometry is often used to avoid plate-plate alignment problems (Fig. 1) [7-10], the Casimir force $F_{\mathrm{C}}$ is given by $F_{\mathrm{C}}=(2 \pi R / A) E_{\mathrm{pp}}$ (assuming $\left.R \gg L\right)$. The material optical properties enter the Lifshitz formula via the frequency dependent dielectric function $\varepsilon(\omega)$. In Eq. (2) the reflection coefficients for $\mathrm{s}$ and p polarization and the function $\varepsilon(\mathrm{i} \zeta)$ are given, respectively, by

$$
\begin{aligned}
& r_{\mathrm{s}}=\frac{k_{\mathrm{o}}-k_{1}}{k_{\mathrm{o}}+k_{1}} \text { and } r_{\mathrm{p}}=\frac{\varepsilon(\mathrm{i} \zeta) k_{\mathrm{o}}-k_{1}}{\varepsilon(\mathrm{i} \zeta) k_{\mathrm{o}}+k_{1}}, \\
& \varepsilon(\mathrm{i} \zeta)=1+(2 / \pi) \int_{0}^{\infty} \frac{\omega \varepsilon "(\omega)}{\omega^{2}+\zeta^{2}} \mathrm{~d} \omega,
\end{aligned}
$$

with $k_{\mathrm{o}}=\sqrt{\zeta^{2} / c^{2}+k^{2}}, k_{1}=\sqrt{\varepsilon(\mathrm{i} \zeta) \zeta^{2} / c^{2}+k^{2}}$, and $\varepsilon^{\prime \prime}(\omega)$ the imaginary part of $\varepsilon(\omega)$.

\section{Experimental procedure}

Polysterene spheres with a diameter of $100 \mu \mathrm{m}$ and a $1.4 \%$-deviation from sphericity were glued on a $450 \mu \mathrm{m}$ long Au coated cantilever (Fig. 1). A relatively stiff cantilever is used to reduce the jump to contact, cantilever bending due to Au evaporation, and errors in the deflection correction. AFM was used to determine the sphere roughness (prior to $\mathrm{Au}$ coating), which gave a $1.2 \mathrm{~nm}$ RMS roughness amplitude over an area of $25 \mu \mathrm{m}^{2}$. Further, the spheres were plasma sputtered for electrical contact with the cantilever, and then were coated with $100 \mathrm{~nm} \mathrm{Au}$ at a rate of $0.6 \mathrm{~nm} / \mathrm{sec}$ in an evaporator kept at a base pressure of $10^{-6}$ mbar. Si-oxide wafers were used as substrates and coated by Au layers of thicknesses between 100-1600 nm, and under identical growth conditions as the Au coating on the sphere (see Fig. 2).

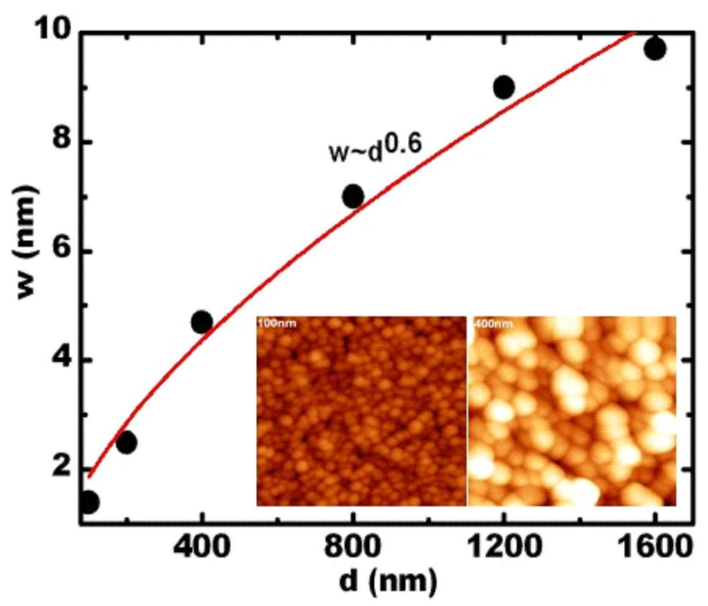

Fig. 2. Increasing roughness amplitude $w$ with increasing film thickness $d$. The inset shows topography examples of two films $100 \mathrm{~nm}$ and $400 \mathrm{~nm}$ thick, respectively.

\section{Van der Waals/Casimir force measurements: effect of surface roughness and optical properties}

The Lifshitz formula accounts for real optical properties of the materials, and for finite temperature effects. Corrections to the force due to optical properties can be very large, especially at small distances $(<100 \mathrm{~nm})$ between the bodies $[7,8]$. An additional source of corrections to the force is the surface roughness $[9,10]$. Roughness corrections $\delta E_{\mathrm{pp}}$ to the Casimir energy (and thus to the force) within the scattering formalism [9] are formulated in terms of a roughness response function $G(k)$ and the roughness power spectrum $\sigma(k)$ :

$$
\delta E_{\mathrm{pp}}=\int\left[\mathrm{d}^{2} k / 4 \pi^{2}\right] G(k) \sigma(k),
$$

where $G(k)$ is derived in [9], yielding for the total energy $E_{\mathrm{pp}, \text { rough }}=E_{\mathrm{pp}}+\delta E_{\mathrm{pp}}$. The theory is valid under the assumptions: first, the lateral dimensions of the roughness must be much smaller than the system size, i.e., plate or sphere, which is usually the case; second, the RMS roughness $w$ must be small compared to the separation distance $L(w \ll L)$; and third, the local surface slope of the film must be small $\left(\rho_{\text {rms }} \ll 1\right)[11]$.

A wide variety of surfaces exhibits the so-called self-affine roughness [16], which is characterized for 
isotropic surfaces by the RMS roughness amplitude $w=$ $\left\langle[h(r)]^{2}\right\rangle^{1 / 2}(\langle h\rangle=0)$, the lateral correlation length $\xi$ (indicating the lateral feature size), and the roughness exponent $0<H<1$. Small values of $H \approx 0$ correspond to jagged surfaces, while large values $H \approx 1$ to a smooth hill-valley morphology [12]. For self-affine roughness the spectrum $\sigma(k)$ scales as $\sigma(k) \propto k^{-2-2 H}$ if $k \xi \gg 1$, and $\sigma(k) \propto$ const if $k \xi \ll 1[12]$. This scaling is satisfied by the analytic model [13]

$$
\sigma(k)=\left(A H w^{2} \xi^{2}\right) /\left(1+k^{2} \xi^{2}\right)^{1+H}
$$

with $A=2 /\left[1-\left(1+k_{\mathrm{c}}^{2} \xi^{2}\right)^{-H}\right], k_{\mathrm{c}}$ is a lower roughness cut-off $\left(\approx 1 \mathrm{~nm}^{-1}\right)$. The parameters $w, \xi$ and $H$ can be determined by direct measurement of the height difference correlation function $H(r)=\left\langle[h(r)-h(0)]^{2}\right\rangle$ with $\langle\ldots\rangle$ denoting the ensemble average over multiple surface scans [13].

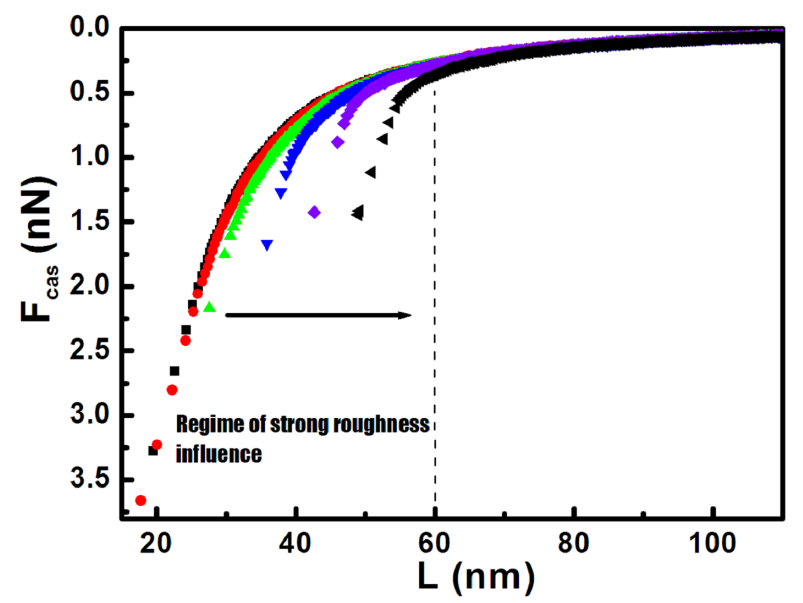

Fig. 3. Force measurements with increasing roughness amplitude $w$ (see Fig. 2) as the arrow shows. The roughness influence becomes evident for sphere-plate separations $L<70 \mathrm{~nm}$.

Furthermore, the Picoforce AFM from Veeco [10] was used for the Casimir force measurements (Fig. 3) following the procedure outlined in [5]. Moreover, averaging over 30 force curves was used when the Casimir force was measured. The plate-sphere separation $L=$ $D_{\text {piezo }}+d_{0}-d_{\text {defl }}$ was measured with respect to the point of contact with the surface, where $D_{\text {piezo }}$ is the piezo movement, $d_{0}$ is the distance upon contact due to substrate and sphere roughness, and $d_{\text {deff }}$ is the cantilever deflection correction $[5,10]$.

The cantilever spring constant $k$ was determined electrostatically with an error of $3 \%$ [10], and it was recalibrated for all films without any measurable roughness effect on the electrostatic force. The residual or contact potential $V_{\mathrm{c}}$ on the grounded sphere was determined electrostatically, and it was found to be $V_{\mathrm{c}} \leq 25 \mathrm{mV}$ (error $\leq 5 \mathrm{mV}$ ) [10]. Once $k$ and $V_{\mathrm{c}}$ are known, $d_{0}$ is determined also electrostatically [10]. Indeed, the $d_{0}$ was found to vary almost linearly with the sum of the RMS roughness amplitudes of the sphere $\left(w_{\mathrm{sph}}\right)$ and substrate $(w)$ : $d_{0} \approx c\left(w+w_{\mathrm{sph}}\right)$ with $c=3.7 \pm 0.3$ [10]. The magnitude of the measured roughness effect on the Casimir force is of the order of $100 \%$ for thicker films (which are also rougher films) at short separations $(<80 \mathrm{~nm})$, while at larger separations the scaling law is recovered and agreement with theory is restored.

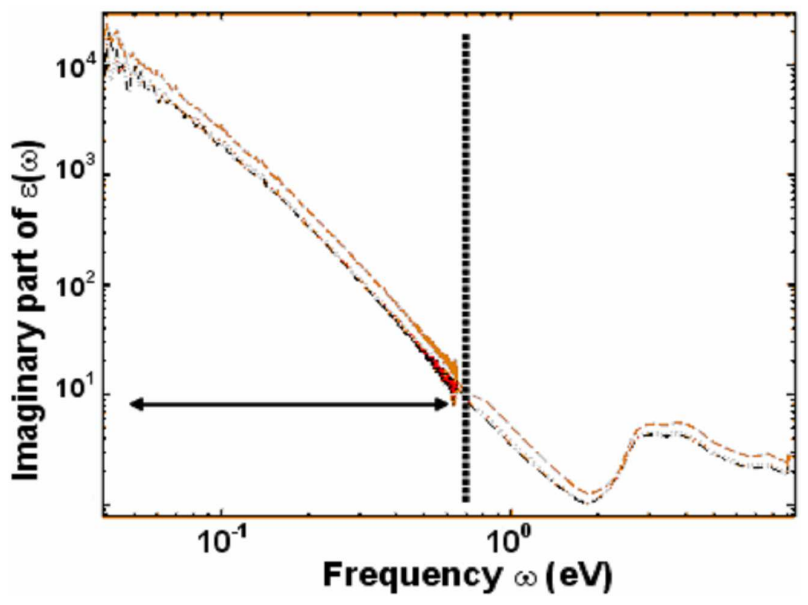

Fig. 4. Ellipsometry measurements of the dielectric function where it is shown its imaginary part. The latter is also relevant for the force measurement with the IR (infrared) - regime indicated behind the dotted line. At high frequences data were extrapolated from handbook data (this regime does not play any significant role in force calculations).

Besides surface roughness the influence of the material optical properties is very crucial. The optical data were obtained from ellipsometry measurements by Woolam for wavelengths $137 \mathrm{~nm}$ to $33 \mu \mathrm{m}$ (see Fig. 4). At low frequences, where the dielectric function is large and gives the highest contribution to the force (Eq. (3)), it was obtained by extrapolation using the Drude model $[7,8]$

$$
\varepsilon(\omega)=1-\omega_{\mathrm{p}}^{2}\left[\omega\left(\omega+\mathrm{j} \omega_{\mathrm{t}}\right)\right]^{-1}
$$

with $\omega_{\mathrm{p}}$ the plasma frequency and $\omega_{\mathrm{t}}$ the relaxation frequency. Furthermore, Fig. 5 shows the theoretical force calculations using the sphere-plate configuration and the optical data of Fig. 4. The data were calibrated with respect to the perfect gold films (single crystal without defects) with $\omega_{\mathrm{p}}=9.0 \mathrm{eV}$ and $\omega_{\mathrm{t}}=0.035 \mathrm{eV}$. The value of $\omega_{\mathrm{p}}=9.0 \mathrm{eV}$ was calculated using $\omega_{\mathrm{p}}=\sqrt{N e^{2} / \varepsilon_{\mathrm{o}} m_{\mathrm{e}}}$ $[7,9]$ with $N$ the number of conduction electrons per unit volume (computed from its bulk density), $e$ the electron charge, and $m_{\mathrm{e}}$ the effective electron mass.

However, the films used for measurement of the Casimir force can contain defects (grain boundaries, impurities etc.), which are responsible for the reduction of $\omega_{\mathrm{p}}(<9.0 \mathrm{eV})[7,8]$. There is significant difference between this reference force curve and those that corresponds to actual Au films as it is shown in Fig. 5. The deviations remain significant even for separations as large as $1000 \mathrm{~nm}\left(\gg \lambda_{\mathrm{p}}=\omega_{\mathrm{p}} / c\right.$; with $\lambda_{\mathrm{p}}$ the plasma wavelength). 


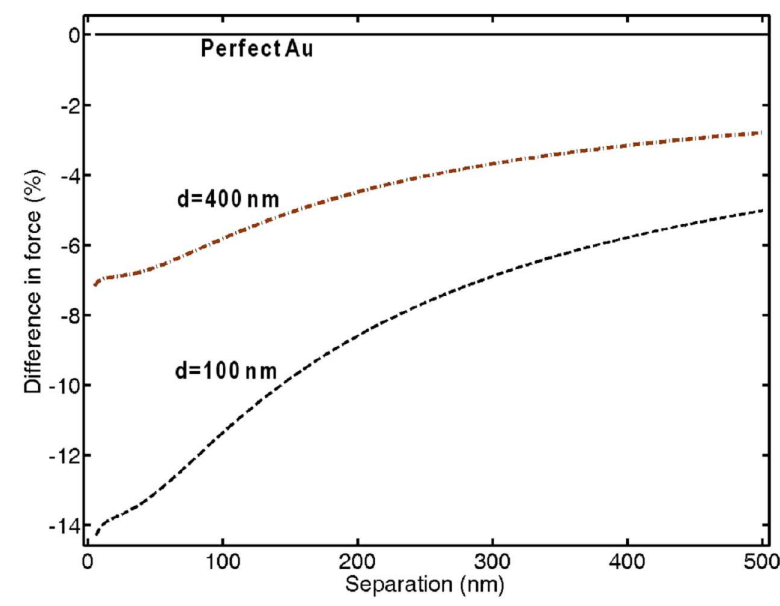

Fig. 5. Force calculations using the optical data from Fig. 4 and Eq. (2). The vertical axis shows the force relative difference (in \%) with respect to the force for perfect $\mathrm{Au}$ films.

\section{Capillary forces}

Finally, we will compare the measured vdW/Casimir forces to capillary adhesive forces. Adhesive forces were measured by retracting a sphere attached on the cantilever from the surface. The total adhesion force can be divided into a capillary force and an interfacial tension force due to surface tension acting tangentially to the interface along the contact line with the solid body [14]. For the relatively smooth films, the theory prediction for flat surfaces gives an approximate upper limit for the force $F_{\text {up }}=4 \pi \gamma R_{\mathrm{s}} \cos (\theta)$ [14] $\left(F_{\text {up }}=S \Delta \sigma\right.$ with $S=$ $4 \pi R_{\mathrm{s}} R_{\mathrm{k}} \cos (\theta)$ the meniscus surface area and $\Delta \sigma=\gamma / R_{\mathrm{k}}$ the Laplace pressure, while ignoring contributions from surface tension).

For the contact angle of water onto $\mathrm{Au}$ surfaces of approximately 70 degrees and $R_{\mathrm{s}}=50 \mu \mathrm{m}$, we obtain the adhesive force $F_{\text {up }}=1.5 \times 10^{4} \mathrm{nN}$ [15]. With increasing roughness, surfaces can be more hydrophobic [16] leading to reduced capilary force. In addition, the contact area between sphere and plate is highly reduced. If we consider the lower limit for the force in terms of contact onto a single asperity [15], where the capillary meniscus is formed only between this asperity and the surface of the sphere, we obtain a force of magnitude $F_{\text {low }}=4 \pi \gamma \xi \cos (\theta)=15 \mathrm{nN}$ (where we used as an effective asperity radius the roughness correlation length $\xi$ ). From Fig. 6 it appears that the smooth limit is reached for the $\mathrm{Au} /$ mica film. For the roughest films the values found are up to ten times higher than that of a single asperity indicating a capillary interaction of a multitude of asperities. In any case, as Fig. 6 indicates, the effect of capillary forces can be by orders of magnitude larger than that of vdW/Casimir forces. Nevertheless, they have also a high sensitivity on changes of surface roughness.

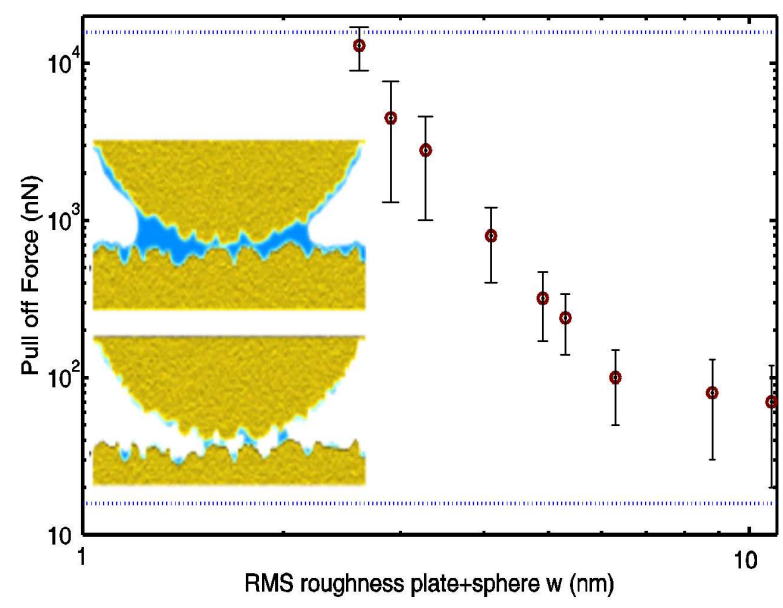

Fig. 6. Adhesion force vs. RMS roughness of the sphere and plate added together. The upper and lower horizontal lines represent the theoretical values for capillary interaction, in case of a perfectly smooth sphere with a perfectly smooth plate, and in case of a single asperity with lateral size $50 \mathrm{~nm}$. The inset shows complete (upper) and multiple asperity (lower) meniscus formation.

\section{Conclusions}

In conclusion, surface functionality with respect to its influence on the Casimir force is strongly affected by surface morphology and material optical properties. The effect of capillary forces can be by orders of magnitude larger than that of vdW/Casimir forces. Nevertheless, they have also a high sensitivity on changes of surface roughness. Therefore, realistic implementations of these forces in MEMS/NEMS should take carefully into account the associated morphology and material functionality.

\section{Acknowledgments}

The research was carried out under project number MC3.05242 in the framework of the Strategic Research programme of the Materials innovation institute M2i (the former Netherlands Institute for Metals Research or NIMR). Financial support from the M2i is gratefully acknowledged. We would like to acknowledge useful discussions with V.B. Svetovoy.

\section{References}

[1] H.B.G. Casimir, Proc. K. Ned. Akad. Wet. 51, 793 (1948). For initial measurements of the Casimir effect see: M.J. Sparnaay, Physica (Utrecht) 24, 751 (1958); P.H.G.M. van Blockland, J.T.G. Overbeek, J. Chem. Soc. Faraday Trans. 74, 2637 (1978). 
[2] F.M. Serry, D. Walliser, G.J. Maclay, J. Appl. Phys. 84, 2501 (1997); H.B. Chan, V.A. Aksyuk, R.N. Kleiman, D.J. Bishop, F. Capasso, Science 291, 1941 (2001); G. Palasantzas, J.Th.M. De Hosson, Phys. Rev. B 72, 115426 (2005); ibid Phys. Rev. B 72, 121409 (2005); G. Palasantzas, J.Th.M. De Hosson, Surf. Sci. 600, 1450 (2006).

[3] R. Onofrio, New J. Phys. 8, 237 (2006).

[4] S.K. Lamoreaux, Phys. Rev. Lett 78, 5 (1997); S.K. Lamoreaux, Phys. Rev. Lett. 83, 3340 (1999); S.K. Lamoreaux, Phys. Today, (2007).

[5] B.W. Harris, F. Chen, U. Mohideen, Phys. Rev. A 62, 052109 (2000).

[6] E.M. Lifshitz, Zh. Eksp. Teor. Fiz. 29, 94 (1956) [Sov. Phys. JETP 2, 73 (1956)]; I.E. Dzyaloshinskii, E.M. Lifshitz, L.P. Pitaevskii, Advances in Physics 38, 165 (1961); E.M. Lifshitz, L.P. Pitaevskii, Statistical Physics, Part 2, Pergamon Press, Oxford 1980.

[7] I. Pirozhenko, A. Lambrecht, V.B. Svetovoy, New J. Phys. 8, 238 (2006).

[8] V.B. Svetovoy, P.J. van Zwol, G. Palasantzas, J.Th.M. De Hosson, Phys. Rev. B 77, 035439 (2008); P.J. van Zwol, G. Palasantzas, J.Th.M. DeHosson, Appl. Phys. Lett. 91, 144108 (2007).
[9] P.A. Maia-Neto, A. Lambrecht, S. Reynaud, Phys. Rev. A 72, 012115 (2005); P.A. Maia-Neto, A. Lambrecht, S. Reynoud, Europhys. Lett. 69, 924 (2005).

[10] P.J. van Zwol, G. Palasantzas, J.Th.M. De Hosson, to appear in Phys. Rev. B (2008); P.J. van Zwol, G. Palasantzas, M. van de Schootbrugge, J.Th.M. De Hosson, Appl. Phys. Lett. 92, 054101 (2008).

[11] G. Palasantzas, Phys. Rev. E 56, 1254 (1997).

[12] P. Meakin, Phys. Rep. 235, 1991 (1994); J. Krim, G. Palasantzas, Int. J. Mod. Phys. B 9, 599 (1995).

[13] G. Palasantzas, Phys. Rev. B 48, 14472 (1993); 49, 5785 (1994); G. Palasantzas, J. Krim, Phys. Rev. Lett. 73, 3564 (1994).

[14] A. Ata, Y.I. Rabinovich, R.K. Singh, J. Adhesion, Sci. Technol. 16, 337 (2002).

[15] P.J. van Zwol, G. Palasantzas, J.Th.M. de Hosson, Phys. Rev. E 78, 031606 (2008); P.J. van Zwol, G. Palasantzas, J.Th.M. DeHosson, Appl. Phys. Lett. 91, 101905 (2007).

[16] M.E. Abdelsalam, P.N. Bartlett, T. Kelf, J. Baumberg, Langmuir 21, 1753 (2005). 